\title{
Can Transcranial Direct Current Stimulation Improve the Resistance Strength and Decrease the Rating Perceived Scale in Recreational Weight-Training Experience?
}

\author{
Eduardo Lattari, ${ }^{1}$ Maria L. Andrade, ${ }^{1}$ Alberto S. Filho, ${ }^{1}$ Antônio M. Moura, ${ }^{1}$ \\ Geraldo M. Neto, ${ }^{1}$ Júlio G. Silva, ${ }^{2}$ Nuno B. Rocha, ${ }^{3}$ Ti-Fei Yuan, ${ }^{4}$ Oscar Arias-Carrión, ${ }^{5}$ \\ AND SÉrgio Machado ${ }^{1}$ \\ ${ }^{1}$ Physical Activity Sciences Post-Graduate Program (PGCAF), Salgado de Oliveira University (UNIVERSO), Niterói, \\ Brazil; ${ }^{2}$ Rehabilitation Science Masters Program, Augusto Motta University Center (UNISUAM), Rio de Janeiro, Brazil; \\ ${ }^{3}$ School of Allied Health Sciences, Polytechnic Institute of Porto, Porto, Portugal; ${ }^{4}$ School of Psychology, Nanjing Normal \\ University, Nanjing, China; and ${ }^{5}$ University of Trastornos del Movimiento y Sueño (TMS), Hospital General Dr. Manuel \\ Gea Gonzalez, Secretaria de Salud, México, Mexico
}

\begin{abstract}
Lattari, E, Andrade, ML, Filho, AS, Moura, AM, Neto, GM, Silva, JG, Rocha, NB, Yuan, T-F, Arias-Carrión, O, and Machado, S. Can transcranial direct current stimulation improve the resistance strength and decrease the rating perceived scale in recreational weight-training experience? J Strength Cond Res 30 (12): 3381-3387, 2016-The goal of this study was to evaluate the acute efficacy of anodic transcranial direct current stimulation on the total volume of repetitions and perceived exertion in recreationally trained individuals in strength. The sample consisted of 10 participants trained in exercise against resistance for at least 3 months. Participants underwent elbow flexion exercise at barbell with a specific load of 10 repetition maximum (10RM), responded immediately after the OMNIRES scale, and were stimulated for 20 minutes with a tDSC protocol $(2 \mathrm{~mA})$, depending on randomization. After applying the tDSC, subjects were again subjected to perform elbow flexion with 10RM load and, soon after, again responded to OMNI-RES scale. All subjects underwent the 3 experimental conditions of the study, c-tDSC, a-tDSC, and sham-tDSC, which were randomized. A range of 48-72 hours was allowed between each assessment visit. An interaction to condition and time $(F=52.395 ; p \leq 0.001)$ has shown that repetitions completed after anodic condition were higher compared with the other conditions in the postsession. In relation to perceived exertion, verified by OMNI-RES scale, 2-way analysis of variance for repeated measures showed an interaction between
\end{abstract}

Address correspondence to Dr. Sérgio Machado, secm80@Yahoo.com.br. 30(12)/3381-3387

Journal of Strength and Conditioning Research

(C) 2016 National Strength and Conditioning Association condition and time $(F=28.445 ; p \leq 0.001)$, where the perceived exertion was decreased after the a-tDSC condition and increased after the c-tDSC condition. In strict terms of performance, it seems to be beneficial to attend a session of $20 \mathrm{mi}-$ nutes a-tDSC, when strength training practitioners can no longer support high-volume training and have increased responses in the perceived exertion.

KEY WoRD total repetition, tDCS, strength, prefrontal dorsolateral cortex

\section{INTRODUCTION}

$\mathrm{T}$ ranscranial direct current stimulation (tDCS) is a noninvasive neuromodulatory brain stimulation technique that emits a low electric current directly and continuously over the target brain area through electrodes positioned at predetermined locations of the scalp (26). Transcranial direct current stimulation is able to sufficiently modify the neuronal transmembrane potential influencing the excitatory levels and modulating the firing rate of isolated neuronal cells $(2,20,21)$. A continuous electrical current ranging from 0.4 to $2 \mathrm{~mA}$ is imposed on the cortical region for a period of 3-20 minutes, where by means of this procedure, changes in cortical excitability have been achieved (25). Depending on the polarity of selected electrical current, different effects are triggered, the anode (a-tDCS) (i.e., increased cortical excitability of the target regions) and the cathode (c-tDCS) (decreases cortical excitability target regions) (25).

This stimulation technique has been placed into the context of exercise, which results showed that a-tDCS applied to the motor cortex (MC) promoted acute (13) and chronic (14) increases in muscle strength of the contralateral limb. One benefit found using a-tDSC applied to the 
$\mathrm{MC}$ was the ability to sustain a submaximal voluntary contraction (20\% of maximum voluntary contraction-MVCelbow flexors) for longer periods of time (31\%), allowing a greater volume muscle contractions (31). Another important aspect of this type of noninvasive brain stimulation is that it can help on the decrease of perceived exertion at submaximal exercise, and may be capable of modulating the sensory perception of effort (27). Physical fatigue is a complex phenomenon and factors such as perception of effort and central inhibition may be involved (8). When the brain is faced with fatigue situations, there is an increase in beta power (i.e., increased frequency band) and greater coherence between the electrodes of the dorsolateral prefrontal cortex (DLPFC) (F3-F4). These findings suggest the existence of a compensatory mechanism underlying physical performance, managed by the DLPFC, when there is reduced MC activation by the presence of central fatigue induced by effort (24). Among the mechanisms suggested to date, the a-tDSC can seem to show improvements in the relationship between the electric discharge rate of cortical neurons and the strength production (30), reduction of perceived exertion a same submaximal load of work (27), relative inhibition of the alpha motor neurons and modulating central fatigue or muscle pain during exertion (6), and increased cortical excitability induced by a-tDCS (31).

Despite the supposed benefit of a-tDSC on different manifestations of muscle strength and perceived exertion, a study conducted by Kan et al. (16) demonstrated that with this noninvasive stimulation technique, there were no increases in MVC and the ability to sustain longer submaximal voluntary contraction ( $30 \%$ of MVC) by the elbow flexors. In another study, a sustained task of elbow flexion with $20 \%$ of MVC, the a-tDSC generated greater fatigue and increased perception of effort compared with a placebo condition (sham-tDSC) (31). Because the effects of tDCS on the resistance strength and perception of effort seem to be dependent on the site of electrode placement, $\mathrm{MC}$ and Prefrontal cortex (PFC), further studies are needed for further clarification on the matter. Furthermore, it should be noted that most of the effects of tDSC studies reporting on the performance of strength, used isometric submaximal exercise tests $(6,16,19)$. It was possible to locate only 1 study on the effects of tDCS on the maximum strength performance in dynamic exercise (12), however, for their study, they have used a small muscle group (wrist extensors), which is not very commonly used in strength training. However, comparisons between the effects of neuromodulation in the MC by anode, cathode, and sham-tDSC on the submaximal strength performance during dynamic submaximal exercise remain scarce. There are, therefore, important knowledge gaps to be filled, especially with regard to the elucidation of the real effects of induction possibilities facilitatory by tDCS technique on the ability to sustain submaximal dynamic muscle contractions and lower perceived exertion. In this context, it is understood that the a-tDSC can be an effective and new tool to allow a greater volume of submaximal muscle contractions and reduction of perceived exertion in recreational practitioners of strength training.

The goal of this study was to evaluate the acute efficacy of a-tDCS on the total volume of repetitions and perceived exertion in intermediate training experience lifters in strength. Because of the t-DSC anode induces increased cortical excitability and reduction of perceived exertion a same submaximal load of work, our hypothesis is that the a-tDCS will cause greater total volume of repetitions and decreases the perceived exertion when comparing with the conditions c-tDCS and sham-tDSC.

\section{Methods}

\section{Experimental Approach to the Problem}

The participants were all university students who volunteered to the study. Each participant visited the laboratory 6 times. On the first visit, a medical history, a questionnaire to verify the physical activity level of each subject, and a familiarization to test 10 repetition maximum (10RM), and the scale of perceived exertion were conducted. A week after the familiarization process, the subjects performed a 10RM test and 48 hours after this, a retest 10RM. In the 3 subsequent visits, participants were initially placed in a quiet room for 10 minutes and then the 10RM test was applied, followed immediately by the application of tDCS anode, cathode, or sham ( $2 \mathrm{~mA}$ for 20 minutes). Immediately after applying the tDCS, the subjects performed a total volume of repetitions with the load used in the 10RM test. The same procedures were performed in 3 visits and were separated by $48-72$ hours in a randomized design. The total volume of repetitions and rating perceived exertion (OMNI-RES) were examined before and after each experimental condition: anodal-tDCS (a-tDCS), cathodal-tDCS (c-tDCS), and sham-tDCS (sham-tDSC). The evaluators did not know whether the data corresponded to the a-tDCS anode, c-tDSC, or sham-tDSC. All measures in each visit were performed in the morning (i.e., 8:00-10:00 hour AM) to avoid circadian effects on strength. The ambient temperature ranged from $21^{\circ} \mathrm{C}$ to $23^{\circ} \mathrm{C}$ and relative humidity ranged from 55 to $70 \%$. The subjects received the information to keep their food diet routine normally before performing the visits but were discouraged to consume ergogenic beverages like coffee.

\section{Subjects}

The sample consisted of 10 undergraduate students from the Salgado de Oliveira University, male, aged $26.5 \pm 5$ years, height of $181.2 \pm 2 \mathrm{~cm}$, and $88.2 \pm 4 \mathrm{~kg}$ body weight.

All were right-handed, physically active according to the classification by the International Physical Activity Questionnaire (IPAQ) (22), and trained in exercise against resistance. Only participants classified as intermediate (individuals with approximately 6 months of consistent resistance training experience) to advanced (individuals with years of resistance training experience) were recruited (1). 


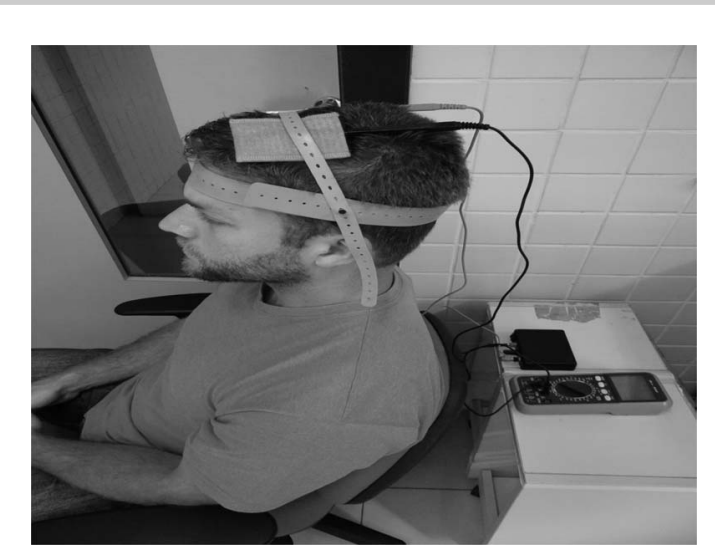

Figure 1. Positioning of the electrodes and assembly of transcranial direct current stimulation.

Participants were excluded if they had any neuropsychiatric, cardiovascular, or osteoarticular disorders, neuropsychiatric use of drugs such as citalopram, amphetamine, L-Dopa, sulpiride and pergolide, lorazepam, rivastigmine, and D-dextromerthorpan cucloserine, carbamazepine and flunarizine and, if they drank any alcohol the day before or any beverage that contains caffeine on the day of the experiment.

All participants were initially informed about the procedures and signed an informed consent according to the Norms for Research with Human Beings (Resolution No. 466/2012 CNS) was given. This project was approved by the Ethics Committee of the Salgado de Oliveira University under the $730.098 / 2014$ protocol number.

\section{Determination of $\mathbf{1 0}$ Maximum Repetition Loads}

All subjects were familiarized with the 10RM test at least a week before testing. Before the determination of the 10 RM load, a warm-up was performed using $50 \%$ of the expected load. To determine the load on the 10RM test, we used the elbow flexion exercise in the free barbell with maximum load, which considering the trial and error system, it offers greater accuracy and precision as the test. There were no more than 3 attempts, with a 5-minute break between them, given that the results could be adversely affected because of the excessive fatigue induced by the high number of repetitions per muscle group (7).

Verbal encouragement was made during strength testing, to improve performance (23). The execution of the movement was cadenced by a metronome (Seiko/DM-50) consisting of the period of 2 seconds per phase of the movement (concentric/eccentric). Finally, subjects were instructed to expire in the concentric phase and to inspire in the eccentric phase. The following strategies have been adopted during the test 10RM, to reduce errors of execution:

(1) All participants were properly instructed about the test procedures and performance technique in elbow flexion exercise with free weight;

(2) In the case of execution error, repetition was not valid;

(3) All tests were performed at the same time for the same individual;

(4) The equipment used for testing and training was properly checked.

The reproducibility of the 10RM test was performed 48 hours after the first test and was analyzed by intraclass correlation coefficient $(\mathrm{ICC}=0.97)$ and typical error of measurement $(\mathrm{TEM}=2.37 \mathrm{~kg} ; 13 \%)$. The load obtained in elbow flexion exercise was $17.5 \pm 3.4 \mathrm{~kg}$. The load 10RM test was used in all conditions, enabling to check the total amount of repetitions that the subjects performed after experimental conditions.

\section{OMNI Perceived Exertion} Scale for Resistance Exercise

The OMNI-RES was used to assess the rating of perceived exertion on the elbow flexor exercise (28). The scale has both verbal and mode-specific pictorial descriptors across a numerical response and narrow range from 0 to 10 . All subjects were made familiar with the scale 1 week before the experimental conditions.

\section{Application of Transcranial Direct Current Stimulation}

The subjects remained seated comfortably in a chair located within the laboratory. The
Figure 2. *Significant interactions between condition and moment. tDCS $=$ transcranial direct current stimulation; a-tDCS $=$ anode-tDCS; c-tDCS $=$ cathode-tDCS. 


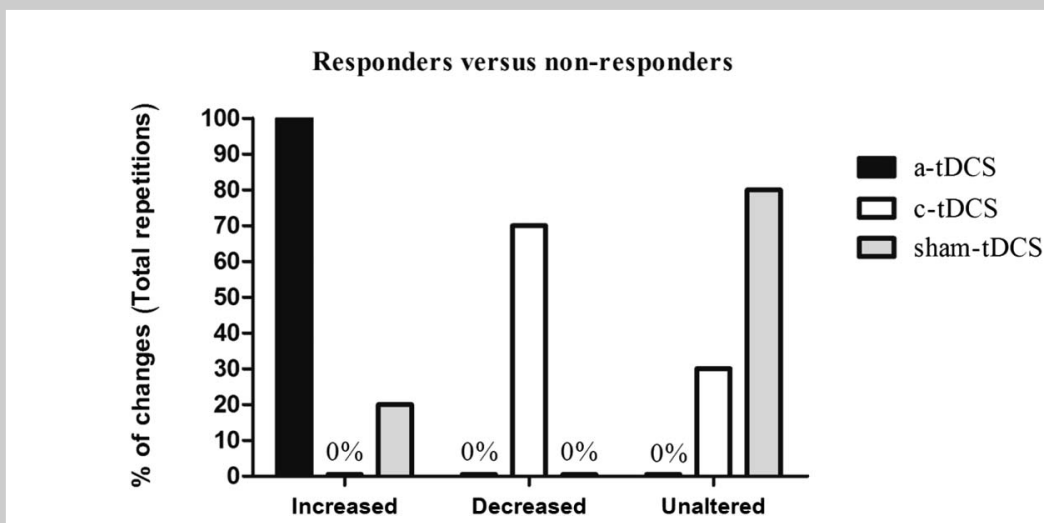

Figure 3. Interindividual variability of responders vs. nonresponders for total repetitions. $t D C S=$ transcranial direct current stimulation; a-tDCS = anode-tDCS; c-tDCS = cathode-tDCS.

(15), whereas the anodic electrode will be placed on the right Orbitofrontal cortex (OBF) (Figure 1).

For placebo condition (sham-tDSC), the electrodes were placed in the same position of the anode stimulation. However, the stimulator was turned off after 30 seconds, which has been reported as ineffective stimulation (11). According to previous studies, with this form of stimulation placebo, patients usually report tingling sensations or itching from the initial electrical stimulation, but do not receive any

electric current of $2 \mathrm{~mA}$ was applied using a pair of pads soaked in saline $(\mathrm{NaCl} 140 \mathrm{mmol}$ dissolved in Milli-Q water) comprising the 2 electrodes $\left(35 \mathrm{~cm}^{2}\right)(26)$. The electrodes (anode and cathode) were connected to a continuous current stimulation device with 3 batteries $(9 \mathrm{~V})$ with a maximum output of $10 \mathrm{~mA}$. The batteries were regulated by a digital multimeter (EZA EZ 984, Hong Kong, China) with a standard error \pm 1.5 . For anodal stimulation (a-tDSC), the anodic electrode was placed in the left DLPFC located in the electrode area F3 in accordance with the international 10-20 system electroencephalogram (EEG) (15). The cathode electrode was placed on the right orbitofrontal cortex, located on the area of the electrode Fp2, both fixed by elastic bands. For cathodal stimulation (c-tDSC), the cathode electrode is placed on the left DLPFC located on electrode area F3 in accordance with the international 10-20 system EEG

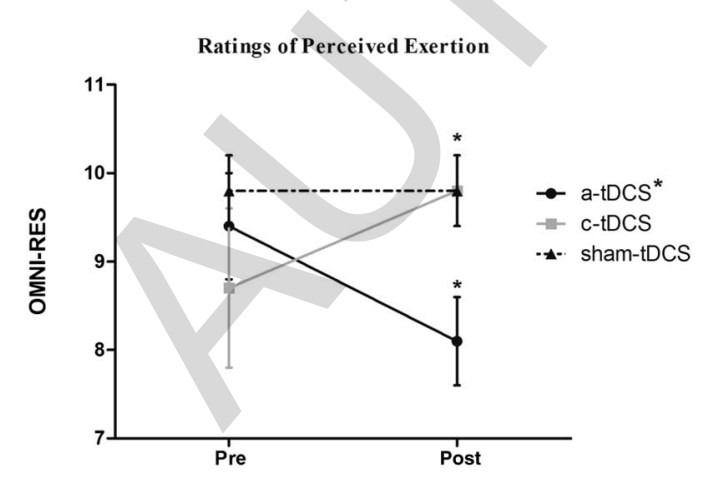

Figure 4. *Significant interactions between condition and moment $(p<0.001)$, OMNI-RES Post $<$ OMNI-RES (a-tDSC) and OMNI-RES Pos $>$ OMNI-RES (c-tDSC); \#main effect for condition, a-tDCS < c-tDCS $(p=0.01)$ and sham-tDCS $(p=0.001)$. tDCS $=$ transcranial direct current stimulation; a-tDCS $=$ anode-tDCS; c-tDCS $=$ cathode-tDCS. other chain. This procedure allows the subjects to become blinded to the type of stimulus that will receive during testing and ensure a control effect (3).

\section{Experimental Procedures}

Data were collected in 6 visits. On the first visit, the participants responded to an interview and to the IPAQ to verify the physical activity level of each participant. After the selection of participants, the consent form was signed by each participant and, soon after, the subjects went through a familiarization to $10 \mathrm{RM}$ test and the scale of perceived exertion (OMNI-RES). On the second visit, a week after the process of familiarization, subjects performed a 10RM test to determine the workload. On the third visit, 48-72 hours after the second one, a new test of $10 \mathrm{RM}$ was performed to verify the reproducibility of the $10 \mathrm{RM}$ load. The fourth to sixth visits, participants underwent elbow flexion exercise at the barbell with a specific load of 10RM, immediately after they answered the OMNI-RES scale and were stimulated for 20 minutes with a tDSC protocols, depending on randomization. After applying the tDSC, subjects were again subjected to perform elbow flexion with 10RM load and, soon after, again replied to the OMNI-RES scale. All subjects underwent the 3 experimental conditions of the study, and c-tDSC-tDSC $2 \mathrm{~mA}$ and sham, which were randomized.

\section{Statistical Analyses}

The normality of the data was performed using the ShapiroWilk test. A 2-way analysis of variance (ANOVA) with repeated measures with 2 entrances, condition $(2 \mathrm{~mA}$ anode vs. $2 \mathrm{~mA}$ cathodic vs. sham) and moment (presession $\times$ postsession) were performed for the total number of repetitions and perceived exertion. When obtaining no effect or interaction, a test post hoc Bonferroni test was used to determine specific differences.

Responders vs. Nonresponders. For interindividual variability, a descriptive analysis was performed using percentage values 
Responders versus non-responders

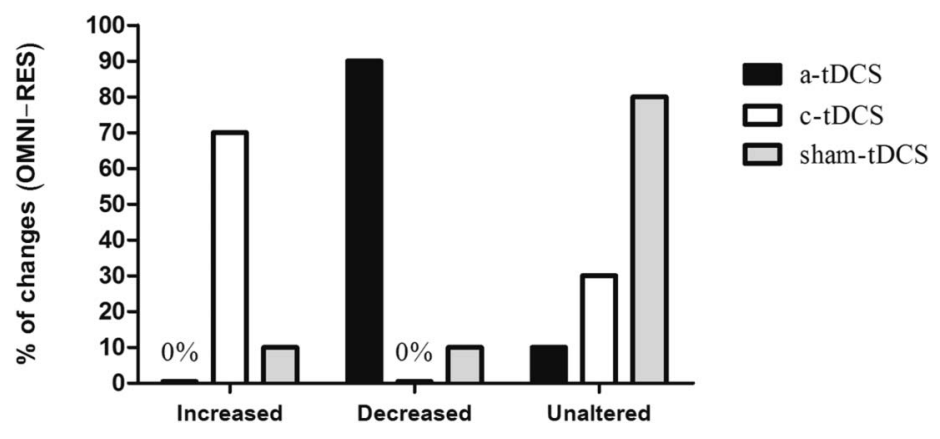

Figure 5. Interindividual variability of responders vs. nonresponders for OMNI-RES. tDCS $=$ transcranial direct current stimulation; a-tDCS $=$ anode-tDCS; c-tDCS $=$ cathode-tDCS.

total volume of repetitions, the ctDSC condition (mean $=9.6$, minimum $=9.3$, and maximum $=9.8 ; p \leq 0.001$ ) and the sham-tDSC condition (mean = 10.1, minimum $=9.9$, and maximum $=10,2 ; p \leq 0.001$ ). The main effect of moment showed that the total volume of repetitions performed after the conditions were higher compared with precondition $(p=$ $0.002)$.

The interindividual variability of responders vs. nonresponders is shown in Figure 3, where a descriptive analysis was performed using the percentage values for individuals

for changes in total repetitions and perceived of exertion from precondition to postcondition.

The magnitude of differences to each condition for total number of repetitions and perceived exertion was established by effect size (ES) analyzes ("d" index), and it calculated by mean differences of moments (pre-post) divided by root square of the sum of standards deviation, and interpreted as suggested by Cohen.

The level of significance was set at $p \leq 0.05$. Data were analyzed using the SPSS software (20.0).

\section{Results}

The 2-way ANOVA for repeated measures showed an interaction between condition and moment $(F=52.395 ; p \leq 0.001)$, the main effect of condition $(F=52.395 ; p \leq 0.001)$, and moment $(F=18.447, p=0.002)$ in the total volume of repetitions completed. An interaction to condition and moment has shown that repetitions completed after anodic condition were higher compared with the other conditions in the postsession (Figure 2). Bonferroni post hoc test showed that the a-tDSC condition $($ mean $=11.1$, minimum $=10.8$, and maximum $=$ 11.4) was higher than the other 2 conditions in relation to the who have increased, decreased, and unaltered the total volume of repetitions.

In relation to perceived exertion, verified by OMNI-RES scale, 2-way ANOVA for repeated measures showed an interaction between condition and moment $(F=28.445 ; p \leq$ $0.001)$ and main effect of condition $(F=18.736$; $p \leq 0.001)$. The post hoc Bonferroni demonstrated an interaction to condition and moment, where the perceived exertion was decreased after the a-tDSC condition and increased after the $\mathrm{c}$-tDSC condition. There was a main effect for condition, where the a-tDSC condition had lower perceived exertion values $($ Mean $=8.7$, Minimum $=8.3$ and Maximum =9.1 $)$ than the sham-tDSC $($ Mean $=9.8$ Minimum $=9.5$ and Maximum $=10$; $p=0.001)$ and $\mathrm{c}-\mathrm{tDSC}$ conditions $($ Mean $=9.2$, Minimum $=8.8$ and Maximum $=9.6 ; p=0.01$ ), respectively (Figure 4).

The inter-individual variability of responders vs. nonresponders is shown in Figure 5, where a descriptive analysis was performed using the percentage values for individuals who have increased, decreased, and unaltered to the ratings perceived exertion.

The magnitude of differences represented by ES from preintervention condition was presented in Table 1.
TABLE 1. Magnitude of differences to each condition for total number of repetitions and perceived exertion.

\begin{tabular}{lccccc}
\hline & \multicolumn{2}{c}{ Effect size $(d)$} & & \multicolumn{2}{c}{ Classification } \\
\cline { 2 - 3 } \cline { 5 - 6 } Condition & Total repetitions & OMNI-RES & & Total repetitions & OMNI-RES \\
\hline a-tDSC & 2.56 & 1.49 & & Very large & Large \\
c-tDSC & 1.26 & 1.23 & & Large & Large \\
Sham-tDSC & 0.47 & 0.47 & & Small & Small \\
\hline
\end{tabular}

\section{Discussion}

The goal of this study was to evaluate the total volume of repetitions and perceived exertion after acute exposure of a-tDCS. It was shown that the a-tDSC condition subjects had a higher volume of elbow flexion contractions when compared with other conditions, c-tDSC and sham-tDSC. Moreover, even with a larger volume of training for the same absolute 
load, the a-tDSC condition, subjects had a lower perception of effort. In c-tDSC condition, there was an increase in the OMNIRES and decrease in the volume of repetitions completed, indicating a reduced resistance strength and increased perceived exertion.

Cogiamanian et al. (6) showed that the endurance time decreased significantly less after anodal $(-21 \%)$ than after cathodal tDCS $(-35 \%)$ or no stimulation $(-39 \%)$, showing that anodal tDCS (tDCS: $1.5 \mathrm{~mA}$ for 10 minutes) over the motor areas of the cerebral cortex improves muscle endurance of the left elbow flexors ( $35 \%$ of MVC). However, in a muscle endurance test with isometric contraction $(30 \%$ of $\mathrm{MVC}$ ) of the elbow flexor, no significant difference in the changes observed between a-tDSC (tDCS: $2 \mathrm{~mA}$ for $10 \mathrm{mi}-$ nutes) and sham sessions (16). In a study by Williams et al. (31), there was no systematic effect of the anodal tDCS stimulation (tDCS: $2 \mathrm{~mA}$ for 20 minutes) on time to task failure of the elbow flexor ( $20 \%$ of MVC) for the entire subjects in set. The subjects were divided into 2 groups related to time to task failure of elbow flexor: full-time, where task failure of elbow flexor occurred before the withdrawal of the tDSC and part-time, where task failure of elbow flexor was extended after withdrawal of the tDSC. Task failure of elbow flexor for the group was full-time $31 \%$ longer with anodal tDSC compared with sham, whereas task failure of elbow flexor for the part-time group did not differ. Moreover, a sustained task of elbow flexion with $20 \%$ of MVC, the a-tDSC generated greater fatigue and increased perception of effort compared with sham condition. For Kan et al. (16), the tDSC does not affect muscle function, perhaps because of the ceiling effect, in which the intervention does not enhance muscle function further when muscle function is already maximal.

Among the several studies that found an increase in strength endurance when using tDSC, only published by Cogiamanian et al. (6) demonstrated favorable results to tDSC anodal. Failure to find any change in the electromyographic variables after tDCS has been suggested by the authors that the tDCS anodal could improve the strength endurance, directly modulating the motor cortical excitability, modulating premotor areas, reducing muscle pain related to fatigue, increase motivation, and improve engagement synergistic muscle (6). However, the use of extremely low loads may have contributed to not significantly altering the electromyographic responses. In the study by Krishnan et al. (17), tDCS anode increased activation in the biceps brachii muscle with $37.5 \%$ and $50 \%$ of MVC, which did not occur at lower power intensities (12.5 and $25 \%$ of MVC). There is the possibility of a greater recruitment and increased rate of fire of the motor units be due to the greater number of sensory inputs sent to the sensorimotor cortex and consequently the MC (4). A further possibility is modulation of the feedback inhibitory systems limiting motor cortical output to "protect" the motor system from overload (6). The pain afferent input from muscle tissue is probably involved in muscle fatigue (10) and tDCS of the MC has been reported to decrease pain (9). The increased cortical excitability after anodal tDCS could therefore depend on a reduced pain sensation arising from muscle tissue during sustained and prolonged voluntary contraction. A study conducted by Tanaka et al. (29) corroborates the results we found. They demonstrated that $\mathrm{a}-\mathrm{tDSC}$ on the MC enhances the physical performance. Morevover, because of the close connection between the MC and the areas responsible for the perceived exertion, such as the cingulate cortex and insular cortex, it leads to the fatigue delay observed. The success of the delay in the perception of effort probably lies in the fact that a-tDSC achieves through existing neural connections, areas that plays a crucial role in central fatigue. These areas are the right DLPFC (Brodmann area 46), the posterior cingulate cortex, and the insular cortex. The right DLPFC is responsible for the activation of sensory motor function areas, to offset the central fatigue (29). For Cogiamanian et al. (6), the a-tDSC slows the sensation of effort because of potentiation of excitement in the premotor areas, and contribute to relationship agonists and antagonists, encourage motivation, and minimize muscle pain. Despite the supposed benefit of a-tDSC on the decrease in perceived of effort, no significant difference was found between the sham-tDSC and in a MVC for the elbow flexion exercise, which is consistent with reports that the effects of tDCS are decreasing excitability during cognitive and motor activities in progress.

These findings require caution because participants of different sexes were used (i.e., men and woman), and specific sex effects have been reported in tDSC (18), as well as on changes in cortical excitability after tDSC (5) and a large variability in muscle function between them. In our findings, the application of a-tDSC increased the total volume of repetitions for all subjects (100\%) and decreased of perceived exertion for $90 \%$ of subjects. This study demonstrated that noninvasive stimulation technique can be effective in strength of resistance and decreased perceived exertion, although that in the placebo condition (sham), no effect was observed for perceived exertion. Nevertheless, the influence of this technique on different types of strength and muscle groups is still unknown, which requires further investigation.

\section{Practical Applications}

This study suggests that the use of a-tDSC has a positive effect on the total volume of repetitions and decrease in perceived exertion. In strict terms of performance, appears to be beneficial to attend a session of 20 minutes a-tDSC when strength training practitioners can no longer support high-volume training and have increased responses in the perceived exertion. Most literature in this area normally uses small muscle groups and isometric contractions, the fact that our study used an elbow flexor exercise, very 
commonly performed in gyms environments and with dynamic contractions, represents a breakthrough in this area. It becomes difficult a correct representation of the cortical area of large muscle groups so that research can move forward in this direction; it requires larger additional information for the correct application of the procedures before strength training.

\section{Acknowledgments}

The authors would especially like to thank the reviewers for their important suggestions. The authors declare that they have no conflicts of interest. No external financial support was required for this project.

\section{REFERENCES}

1. American College of Sports M. American College of Sports Medicine position stand. Progression models in resistance training for healthy adults. Med Sci Sports Exerc 41: 687-708, 2009.

2. Ardolino, G, Bossi, B, Barbieri, S, and Priori, A. Non-synaptic mechanisms underlie the after-effects of cathodal transcutaneous direct current stimulation of the human brain. J Physiol 568: 653663, 2005.

3. Boggio, PS, Zaghi, S, Lopes, M, and Fregni, F. Modulatory effects of anodal transcranial direct current stimulation on perception and pain thresholds in healthy volunteers. Eur J Neurol 15: 1124-1130, 2008.

4. Cafarelli, E and Bigland-Ritchie, B. Sensation of static force in muscles of different length. Exp Neurol 65: 511-525, 1979.

5. Chaieb, L, Antal, A, and Paulus, W. Gender-specific modulation of short-term neuroplasticity in the visual cortex induced by transcranial direct current stimulation. Vis Neurosci 25: 77-81, 2008.

6. Cogiamanian, F, Marceglia, S, Ardolino, G, Barbieri, S, and Priori, A. Improved isometric force endurance after transcranial direct current stimulation over the human motor cortical areas. Eur J Neurosci 26: 242-249, 2007.

7. de Salles, BF, Simao, R, Miranda, F, Novaes Jda, S, Lemos, A, and Willardson, JM. Rest interval between sets in strength training. Sports Med 39: 765-777, 2009.

8. Dundas, JE, Thickbroom, GW, and Mastaglia, FL. Perception of comfort during transcranial DC stimulation: Effect of $\mathrm{NaCl}$ solution concentration applied to sponge electrodes. Clin Neurophysiol 118: 1166-1170, 2007.

9. Fregni, F, Gimenes, R, Valle, AC, Ferreira, MJ, Rocha, RR, Natalle, L, Bravo, R, Rigonatti, SP, Freedman, SD, Nitsche, MA, Pascual-Leone, A, and Boggio, PS. A randomized, sham-controlled, proof of principle study of transcranial direct current stimulation for the treatment of pain in fibromyalgia. Arthritis Rheum 54: 3988$3998,2006$.

10. Gandevia, SC. Spinal and supraspinal factors in human muscle fatigue. Physiol Rev 81: 1725-1789, 2001.

11. Gandiga, PC, Hummel, FC, and Cohen, LG. Transcranial DC stimulation (tDCS): A tool for double-blind sham-controlled clinical studies in brain stimulation. Clin Neurophysiol 117: 845-850, 2006.

12. Hendy, AM and Kidgell, DJ. Anodal tDCS applied during strength training enhances motor cortical plasticity. Med Sci Sports Exerc 45: 1721-1729, 2013.

13. Hendy, AM and Kidgell, DJ. Anodal-tDCS applied during unilateral strength training increases strength and corticospinal excitability in the untrained homologous muscle. Exp Brain Res 232: 3243-3252, 2014.
14. Hendy, AM, Teo, WP, and Kidgell, DJ. Anodal transcranial direct current stimulation prolongs the cross-education of strength and corticomotor plasticity. Med Sci Sports Exerc 47: 1788-1797, 2015.

15. Jasper, H. Progress and problems in brain research. J Mt Sinai Hosp NY 25: 244-253, 1958.

16. Kan, B, Dundas, JE, and Nosaka, K. Effect of transcranial direct current stimulation on elbow flexor maximal voluntary isometric strength and endurance. Appl Physiol Nutr Metab 38: 734-739, 2013.

17. Krishnan, C, Ranganathan, R, Kantak, SS, Dhaher, YY, and Rymer, WZ. Anodal transcranial direct current stimulation alters elbow flexor muscle recruitment strategies. Brain Stimul 7: 443-450, 2014.

18. Kuo, MF, Paulus, W, and Nitsche, MA. Sex differences in cortical neuroplasticity in humans. Neuroreport 17: 1703-1707, 2006.

19. Lampropoulou, SI and Nowicky, AV. The effect of transcranial direct current stimulation on perception of effort in an isolated isometric elbow flexion task. Mot Control 17: 412-426, 2013.

20. Lang, N, Nitsche, MA, Paulus, W, Rothwell, JC, and Lemon, RN Effects of transcranial direct current stimulation over the human motor cortex on corticospinal and transcallosal excitability. Exp Brain Res 156: 439-443, 2004.

21. Lang, N, Siebner, HR, Ward, NS, Lee, L, Nitsche, MA, Paulus, W, Rothwell, JC, Lemon, RN, and Frackowiak, RS. How does transcranial DC stimulation of the primary motor cortex alter regional neuronal activity in the human brain?. Eur J Neurosci 22: 495-504, 2005.

22. Matsudo, S, Araújo, T, Marsudo, V, Andrade, D, Andrade, E, and Braggion, G. International Physical Activity Questionnaire (IPAQ): Study of validity and reproducibility in Brazil. Rev Bras Ativ Fis Saúde 6: 05-18, 2001.

23. McNair, PJ, Depledge, J, Brettkelly, M, and Stanley, SN. Verbal encouragement: Effects on maximum effort voluntary muscle action. Br J Sports Med 30: 243-245, 1996.

24. Menotti, F, Berchicci, M, Di Russo, F, Damiani, A, Vitelli, S, and Macaluso, A. The role of the prefrontal cortex in the development of muscle fatigue in Charcot-Marie-Tooth 1A patients. Neuromuscul Disord 24: 516-523, 2014.

25. Nitsche, MA, Liebetanz, D, Tergau, F, and Paulus, W. [Modulation of cortical excitability by transcranial direct current stimulation]. Der Nervenarzt 73: 332-335, 2002.

26. Nitsche, MA and Paulus, W. Excitability changes induced in the human motor cortex by weak transcranial direct current stimulation. J Physiol 527: 633-639, 2000.

27. Okano, AH, Fontes, EB, Montenegro, RA, Farinatti Pde, T, Cyrino, ES, Li, LM, Bikson, M, and Noakes, TD. Brain stimulation modulates the autonomic nervous system, rating of perceived exertion and performance during maximal exercise. Br J Sports Med 49: 1213-1218, 2015.

28. Robertson, RJ, Goss, FL, Rutkowski, J, Lenz, B, Dixon, C, Timmer, J, Frazee, K, Dube, J, and Andreacci, J. Concurrent validation of the OMNI perceived exertion scale for resistance exercise. Med Sci Sports Exerc 35: 333-341, 2003.

29. Tanaka, S, Hanakawa, T, Honda, M, and Watanabe, K. Enhancement of pinch force in the lower leg by anodal transcranial direct current stimulation. Exp Brain Res 196: 459-465, 2009.

30. Tanaka, S, Takeda, K, Otaka, Y, Kita, K, Osu, R, Honda, M, Sadato, N, Hanakawa, T, and Watanabe, K. Single session of transcranial direct current stimulation transiently increases knee extensor force in patients with hemiparetic stroke. Neurorehabil Neural Repair 25: 565-569, 2011.

31. Williams, PS, Hoffman, RL, and Clark, BC. Preliminary evidence that anodal transcranial direct current stimulation enhances time to task failure of a sustained submaximal contraction. PloS One 8: e81418, 2013. 